\title{
Osteoprotegerin Polymorphisms in a Mexican Population with Rheumatoid Arthritis and Generalized Osteoporosis: A Preliminary Report
}

\author{
Maria Guadalupe Zavala-Cerna, ${ }^{1}$ Maria Cristina Moran-Moguel, ${ }^{2}$ \\ Jesus Alejandro Cornejo-Toledo, ${ }^{2}$ Norma Guadalupe Gonzalez-Montoya, ${ }^{3}$ \\ Jose Sanchez-Corona, ${ }^{2}$ Mario Salazar-Paramo, ${ }^{4}$ \\ Arnulfo Hernan Nava-Zavala, ${ }^{1,5,6}$ Erika Anita Aguilar-Chavez, \\ Miriam Fabiola Alcaraz-Lopez, ${ }^{8}$ Alicia Guadalupe Gonzalez-Sanchez, ${ }^{3}$ \\ Laura Gonzalez-Lopez, ${ }^{9}$ and Jorge Ivan Gamez-Nava ${ }^{10}$ \\ ${ }^{1}$ Programa Internacional, Facultad de Medicina, Universidad Autonoma de Guadalajara, 44100 Zapopan, JAL, Mexico \\ ${ }^{2}$ Division de Medicina Molecular del Centro de Investigacion Biomedica de Occidente, Instituto Mexicano del Seguro Social, \\ 44340 Guadalajara, JAL, Mexico \\ ${ }^{3}$ Programa de Becarios en Investigacion del Instituto Mexicano del Seguro Social, Programa de Doctorado en Farmacologia, \\ Centro Universitario de Ciencias de la Salud, Universidad de Guadalajara, 44340 Guadalajara, JAL, Mexico \\ ${ }^{4}$ Division de Investigacion en Salud, UMAE, Hospital de Especialidades, CMNO, IMSS and Departamento de Fisiologia, \\ CUCS, Universidad de Guadalajara, 44340 Guadalajara, JAL, Mexico \\ ${ }^{5}$ Unidad de Investigacion en Epidemiologia Clinica, Hospital de Especialidades, Centro Medico Nacional de Occidente del Instituto \\ Mexicano del Seguro Social, 44340 Guadalajara, JAL, Mexico \\ ${ }^{6}$ Servicio de Medicina Interna, Inmunologia y Reumatologia, Hospital General de Occidente, Secretaria de Salud Jalisco, \\ 45170 Guadalajara, JAL, Mexico \\ ${ }^{7}$ Unidad de Medicina Familiar 2, Instituto Mexicano del Seguro Social, 44340 Guadalajara, JAL, Mexico \\ ${ }^{8}$ Departamento de Medicina Interna-Reumatologia, Hospital General de Zona 14, Instituto Mexicano del Seguro Social, \\ 44860 Guadalajara, JAL, Mexico \\ ${ }^{9}$ Departamento de Medicina Interna-Reumatologia del Hospital General Regional No. 110, Instituto Mexicano del Seguro \\ Social y Programa de Doctorado en Salud Publica, CUCS, Universidad de Guadalajara, 44340 Guadalajara, JAL, Mexico \\ ${ }^{10}$ Unidad de Investigación en Epidemiologia Clinica, Hospital de Especialidades, Centro Medico Nacional de Occidente \\ del Instituto Mexicano del Seguro Social y Programa de Doctorado en Salud Publica, CUCS, Universidad de Guadalajara, \\ 44340 Guadalajara, JAL, Mexico
}

Correspondence should be addressed to Jorge Ivan Gamez-Nava; drivangamez@prodigy.net.mx

Received 17 July 2014; Revised 12 September 2014; Accepted 15 September 2014

Academic Editor: Giacomina Brunetti

Copyright ( 2015 Maria Guadalupe Zavala-Cerna et al. This is an open access article distributed under the Creative Commons Attribution License, which permits unrestricted use, distribution, and reproduction in any medium, provided the original work is properly cited.

Bone disease in rheumatoid arthritis (RA) is a complex phenomenon where genetic risk factors have been partially evaluated. The system formed by receptor activator for nuclear factor- $\kappa \mathrm{B}$ (RANK), receptor activator for nuclear factor- $\kappa \mathrm{B}$ ligand (RANKL), and osteoprotegerin (OPG): RANK/RANKL/OPG is a crucial molecular pathway for coupling between osteoblasts and osteoclasts, since OPG is able to inhibit osteoclast differentiation and activation. We aim to evaluate the association between SNPs C950T (rs2073617), C209T (rs3134069), T245G (rs3134070) in the TNFRSF11B (OPG) gene, and osteoporosis in RA. We included 81 women with RA and 52 healthy subjects in a cross-sectional study, genotyped them, and measured bone mineral density (BMD) at the lumbar spine and the femoral neck. Mean age in RA was $50 \pm 12$ with disease duration of $12 \pm 8$ years. According to BMD results, 23 (33.3\%) were normal and 46 (66.7\%) had osteopenia/osteoporosis. We found a higher prevalence of C allele for C950T SNP in RA. 
Polymorphisms C209T and T245G did not reach statistical significance in allele distribution. Further studies including patients from other regions of Latin America with a multicenter design to increase the sample size are required to confirm our findings and elucidate if C950T SNP could be associated with osteoporosis in RA.

\section{Introduction}

Rheumatoid arthritis (RA) is a chronic inflammatory disease that affects synovial joints [1]. Its prevalence in Mexico is considerably elevated ranging from 0.3 to $1 \%$ [2]. Currently, the diagnosis of osteoporosis is made on the basis of a measurement by dual-energy x-ray absorptiometry (DXA) at the spine and hip showing a decrement in bone mineral density (BMD) lower than 2.5 standard deviations in comparison with healthy young adults [3]. However, bone involvement in RA is complex; an increased proportion of patients may suffer from osteoporosis; nevertheless causal effects are considered to be multifactorial. Previous studies performed in our center showed a prevalence of axial osteoporosis in Mexican women with RA of $31 \%$ [4].

Genetic risk factors constitute a variable that contributes to influencing the development of osteoporosis in RA, in this context; some genetic polymorphisms may influence the rate of presentation of osteoporosis. Nevertheless such genetic changes do not explain entirely osteoporosis presence or fracture related events. Strong evidence supports the fact that osteoclasts have an important role in osteoporosis and bone erosion formation $[5,6]$.

The system conformed by nuclear factor- $\kappa \mathrm{B}$ (RANK)/ receptor activator for nuclear factor $-\kappa \mathrm{B}$ ligand (RANKL) and osteoprotegerin (OPG) are crucial, for the bone remodeling process. Furthermore, it appeared that not only osteoblasts but also activated $T$ lymphocytes play a crucial role in the pathogenesis of rheumatoid arthritis (RA) and osteoporosis, since they can produce RANKL, which stimulates the differentiation and activation of osteoclasts [7]. These three proteins belong to the superfamily of the TNF- $\alpha$ and have different patterns of expression; RANKL is expressed in osteoblasts and its function is to participate in osteoclasts differentiation $[7,8]$. Osteoprotegerin (OPG) lacks a transmembrane domain, and hence it is a receptor that can be secreted. OPG recognizes and is able to bind RANKL, blocking its interaction with RANK inhibiting osteoclastic differentiation and activation [9-11]. Since OPG is homologous to RANK it acts as a false receptor for RANKL [12-14]. OPG is secreted mainly by stromal cells but can also be secreted by other types of cells [15]. Different studies have been able to identify single nucleotide polymorphisms (SNPs) in TNFSF11 (RANKL) and TNFRSF11B (OPG) genes in association with osteoporosis or low bone density $[12,16]$. It has been documented that OPG overexpression induces an increase in bone density and protects from the development to osteoporosis. In contrast mice that are OPG-deficient develop osteoporosis very rapidly [14, $15,17]$. OPG overexpression blocks osteoclasts maturation; contrariwise, an underexpression of this molecule results in an excessive increase of bone resorption and osteoporosis [18]. Mutations in the promoter region of TNFRSF11B could have influence on the transcription and translation rate of OPG. A study in European population (Eslovenia) showed that two SNPs named rs3134069 and rs3134070 in the TNFRSF11B could form a haplotype with susceptibility to osteoporosis $[16,19]$. To date, there is a lack of information evaluating the relationship between polymorphisms of OPG gene and bone mineral density.

Therefore, the aim of the present study was to assess if there is an association between SNPs C950T (rs2073617), C2097 (rs3134069), and T245G (rs3134070) in the TNFRSF11B (OPG) gene and osteoporosis in rheumatoid arthritis patients.

\section{Patients and Methods}

2.1. Study Subjects. We carried out a cross-sectional study that included 81 consecutive unrelated women who were diagnosed with RA from March 2007 to March 2009. These patients were referred from an outpatient rheumatology clinic in a secondary care center in Guadalajara, Mexico (Hospital General Regional 110, IMSS). Inclusion criteria were as follows: age $\geq 18$ years at entry; self-identified race Mexican Mestizo (defined as individuals who were born in Mexico and were descendants of the original autochthonous inhabitants of the region and of individuals mainly Spaniards); fulfillment of the 1987 American College of Rheumatology (ACR) classification criteria [20]; and no previous BMD measurement. Patients were excluded if pregnancy was present and if they had an overlap syndrome, were receiving bisphosphonates or parathyroid hormone therapy, or had a comorbidity associated with low BMD, such as diabetes mellitus, thyroid disease, or chronic renal failure; patients were not related to each other.

A healthy control group matched by age and sex was obtained from blood donors who attended to "Centro Médico Nacional de Occidente" blood bank; all blood donors were of Mexican Mestizo origin and denied at the time of the study having any disease (acute or chronic).

2.2. Clinical Assessment. Each patient was interviewed using a structured questionnaire to record demographic information, general risk factors for osteoporosis (i.e., age, weight, and height), clinical characteristics, and RA treatment. At the time of the evaluation two trained researchers assessed RA disease activity by systematical evaluation of the DAS28 index [21]; to determine patient disability the Spanish modified version of the Health Assessment QuestionnaireDisability Index (HAQ-DI) [22] was used. Global functional status was evaluated according to the Steinbrocker classification [23]. Rheumatoid factor and C-reactive protein (CRP) were measured by nephelometry using commercially available kits. Erythrocyte sedimentation rate (ESR, mm/h) was measured using the Wintrobe method. 
TABLE 1: Genotyping strategies for TNFRSF11B polymorphism variants detection.

\begin{tabular}{|c|c|c|c|c|c|}
\hline SNP & Primers & Band size (bp) & $\begin{array}{c}\text { Restriction } \\
\text { enzyme }\end{array}$ & $\begin{array}{c}\text { Recognized } \\
\text { sequence }\end{array}$ & $\begin{array}{l}\text { Band size after } \\
\text { digestion (bp) }\end{array}$ \\
\hline \multirow{2}{*}{$\begin{array}{l}\text { C950T } \\
\text { rs2073617 }\end{array}$} & $5^{\prime}$-TGCGTCCGGATCTTGGCTGGATCGG-3' & \multirow{2}{*}{548} & \multirow{2}{*}{ Hinc II } & \multirow{2}{*}{ GTY RAC } & \multirow{2}{*}{$\begin{array}{l}\text { C } 248 \text { and } 287 \\
\text { T } 548\end{array}$} \\
\hline & $5^{\prime}$-ACTTACCACGAGCGCGCAGCACAGCAA-3' & & & & \\
\hline \multirow{2}{*}{$\begin{array}{l}\text { C209T } \\
\text { rs3134070 }\end{array}$} & $5^{\prime}$-CGAACCCTAGAGCAAAGTGC-3' & \multirow{2}{*}{271} & \multirow{2}{*}{ Taq I } & \multirow{2}{*}{ T CGA } & \multirow{2}{*}{$\begin{array}{l}\text { C 212, } 31 \text { and } 28 \\
\text { T } 212 \text { and } 59\end{array}$} \\
\hline & $5^{\prime}$-TGTCTGATTGGCCCTAAAGC-3' & & & & \\
\hline \multirow{2}{*}{$\begin{array}{l}\mathrm{T} 245 \mathrm{G} \\
\text { rs3134069 }\end{array}$} & $5^{\prime}$-CGAACCCTAGAGCAAAGTGC-3' & \multirow{2}{*}{271} & \multirow{2}{*}{$\operatorname{Hinf} I$} & \multirow{2}{*}{ G ANTC } & \multirow{2}{*}{$\begin{array}{l}\text { A } 195 \text {, and } 176 \\
\text { C } 127,76 \text { and } 69\end{array}$} \\
\hline & $5^{\prime}$-TGTCTGATTGGCCCTAAAGC-3' & & & & \\
\hline
\end{tabular}

SNP: single nucleotide polymorphism and bp: base pair.

2.3. BMD Measurement. BMD was measured $\left(\mathrm{g} / \mathrm{cm}^{2}\right)$ by DEXA using a Lunar Prodigy densitometer (GE Medical Systems Lunar, Madison, WI, software V 8.8; GE Medical Systems); the anatomical regions assessed were lumbar spine in the posterior-anterior projection (L1-L4) and femoral neck. The coefficient of variation during the measurement of a standard phantom in our laboratory is $0.7 \%$. The coefficient of variation was $2.4 \%$ at the lumbar spine and $1.6 \%$ at the femoral neck. All scans were performed by the same experienced technician, who was blinded to the characteristics of patients. Each patient was classified into one of the following categories proposed by WHO: normal BMD, defined as having a measurement of BMD within $1 \mathrm{SD}$ of the $\mathrm{BMD}$ of normal young adult women (equivalent to $T$ score $>-1$ ); osteopenia, defined as having a $T$ score in BMD between -1 and $-2.4 \mathrm{SD}$; and osteoporosis, defined as having a $T$ score in BMD $\leq$ -2.5 SD. Patients with a $T$ score $<-1.0$ were considered to have low $\mathrm{BMD}$ in consequence; for analysis purposes in our study we decided to include osteopenia and osteoporosis in one single group denominated as "low BMD".

2.4. Genotyping. DNA from 81 patients with rheumatoid arthritis was extracted from blood samples using conventional methods [24] and stored frozen at $-80^{\circ} \mathrm{C}$.

We chose three SNPs that are present in the promoter region ( $5^{\prime} \mathrm{UTR}$ ) of the TNFRSF11B (OPG) gene; such polymorphisms were previously reported in association with osteoporosis in postmenopausal women or related conditions [19, 25-28]. We performed 3 polymerase chain reactionrestriction fragment length polymorphisms (PCR_RFLPs) protocols as previously described $[26,29,30]$. Table 1 shows primer sequence, products obtained after PCR, restriction enzymes used, their recognition site, and end products after restriction.

Each PCR reaction was carried out in $10 \mu \mathrm{L}$ final volume containing (final concentrations) $1 \mathrm{X}$ buffer $(200 \mathrm{mM}$ Tris$\mathrm{HCl} \mathrm{pH} \mathrm{8.4,} 500 \mathrm{mM} \mathrm{KCl}$, and $4 \mathrm{mM} \mathrm{MgCl}_{2}$ ); $5 \mathrm{pmol} / \mathrm{mL}$ each of the pair primers according to polymorphism (Table 1); $10 \mathrm{mM}$ each of the four deoxyribonucleoside triphosphates; $1 \mathrm{U}$ of taq DNA polymerase (Invitrogen, Carlsbad, CA, USA); and 200 to $300 \mathrm{ng}$ DNA template. The PCR products were visualized by electrophoresis in $10 \%$ polyacrylamide gels at $150 \mathrm{~V}$ for $90 \mathrm{~min}$, followed by silver staining.
2.5. Statistical Analysis. Allelic and genotypic frequencies for each SNP were determined by gene count. Hardy-Weinberg equilibrium was tested, using $\chi^{2}$ test. Genotypic differences between RA patients with and without osteoporosis were evaluated by Mantel-Haenszel test using the EPI INFO (version 6.04d) statistical program. For allelic differences, we used Fisher's exact test. Odds ratios (OR) and 95\% confidence interval $(95 \% \mathrm{CI})$ were computed to evaluate the risk for osteoporosis conferred by presence of the risk alleles. All analyses used two-sided tails with a $P$ value of $\leq 0.05$ used as significance criterion.

\section{Results}

3.1. Demographics and Clinical. We included 81 patients, classified as RA according to ACR classification criteria [20], and 52 healthy subjects. For the RA group, mean age was $50 \pm 12$ years, $100 \%$ of the studied subjects were women, mean duration disease was $12 \pm 8$ years, and all of them were receiving treatment, some of them with monotherapy and others combined therapy; the number and percentages of patients taking a specific drug are listed in Table 2.

RA patients were evaluated for BMD according to the classification criteria of the WHO for osteoporosis. According to the results of bone densitometry 23 patients $(33.3 \%)$ had normal BMD, 31 (44.9\%) had osteopenia (low bone density), and 15 (21.7\%) had osteoporosis. For study purposes we grouped osteopenia with osteoporosis in one group constituted by 46 patients $(66.7 \%)$ (see Table 2$)$. From these, 23 patients (32.9\%) had osteopenia and 13 (18.6\%) had osteoporosis in the lumbar spine, whereas 35 patients $(50.7 \%)$ had osteopenia and $7(10.1 \%)$ had osteoporosis of the femoral neck. Clinical risk factors and factors related to RA severity for low BMD were registered and are listed in Table 2.

3.2. Molecular Analysis. We performed at least three PCRRFLP tests for every subject included in the present study but were able to obtain complete genotyping results in 77 DNA samples from RA patients and 52 healthy subjects. Healthy subjects group was in Hardy-Weinberg equilibrium (data not shown).

In Table 3 genotype and allele frequencies of three SNPs in the OPG gene in healthy subjects and rheumatoid arthritis 
TABLE 2: Clinical characteristics of study patients.

\begin{tabular}{lc}
\hline Characteristic & $n=81$ \\
\hline Age (years), median (range) & $49.9(21-79)$ \\
Menopausal, $n$ (\%) & $43(53.1)$ \\
Weight (kg), median (range) & $65.8(43-94)$ \\
BMI, median (range) & $26.87(18-38)$ \\
Oral contraceptives use, $n$ (\%) & $41(50.6)$ \\
Current smoker, $n$ (\%) & $17(21)$ \\
Family history of fractures $n$ (\%) & $6(7.4)$ \\
Alcohol consumption 3 or more & $3(3.37)$ \\
units/day, $n$ (\%) & $11.8(1.5-35)$ \\
Duration of RA (years), median (range) & $3.8(0-6.7)$ \\
DAS-28 score, median (range) & $7(7.8)$ \\
Global functional status III-IV, $n$ (\%) & $0.7(0-2.8)$ \\
HAQ-DI score, median (range) & $n(\%)$ \\
\hline Medications & $72(88.9)$ \\
\hline Glucocorticoid & $4.4(0-10)$ \\
Prednisone dosage (mg/day), median & $56(70.9)$ \\
(range) & $39(49.4)$ \\
Methotrexate & $27(34.2)$ \\
Sulfasalazine & $25(31.6)$ \\
Chloroquine & $10(12.7)$ \\
Azathioprine & $8(10.1)$ \\
D-Penicillamine & $n(\%)$ \\
Biologic agents & $23(33.3)$ \\
BMD (WHO classification) & $46(66.7)$ \\
\hline Normal & Osteopenia/osteoporosis \\
\hline BMI body mas ing & \\
\hline
\end{tabular}

BMI: body mass index, RA: rheumatoid arthritis, DAS-28: disease activity score, HAQ-DI: health assessment questionnaire-disability index, BMD: bone mineral density, and WHO: World Health Organization.

patients are shown; we found significant difference in the distribution of the C950T (rs2073617) alleles, the C allele being more prevalent in the RA group. Polymorphisms C209T and T245G did not reach statistical significance.

We further analyzed genetic variants in RA patients and divided this group with respect to bone mass index status according to the WHO classification in normal or osteopenia/osteoporosis; there was no significant difference in allele distribution between RA patients with and without osteoporosis; Table 4 shows genotype and allele frequencies in these subgroups.

\section{Discussion}

Osteoporosis represents a major health problem throughout the world and the most serious consequence of osteoporosis is hip fracture, which has a high associated morbidity and mortality [31]. Systemic effects of RA in bone remodeling include loss of axial and appendicular bone mass, associated with an increase in fracture risk ( 9.6 per 1,000 person-years)
[32]. Clinical patterns of bone mass loss include generalized osteoporosis (axial and peripheral), juxta-articular osteoporosis which is adjacent to synovial membrane, and marginal and subchondral erosions which are directly associated with inflamed synovial tissue [33]. A high proportion of patients will suffer from hip and spine fractures causing limitations in physical abilities and social capabilities. Osteoporosis presence in RA patients involves multiple factors, such as disease activity, the use of some prescribed drugs particularly DMARDs and corticosteroids, female sex, older age, and menopause [34].

The development of erosive lesions as well as generalized osteoporosis in RA results from an imbalance between bone mass formation and bone resorption process. In RA the unsteadiness between osteoblastic bone formation (mesenchymal origin) and osteoclastic bone resorption (derived from monocytes/macrophages) promotes bone resorption by osteoclasts and as a consequence loss in bone strength, which may lead to the development of osteoporosis and fractures.

Multiple interactions between bone and the immune system have been described; bone is a major target of chronic inflammation, since it increases bone resorption and results in suppressed local bone formation, causing a wide spectrum of bone involvement in RA [35]. Lymphocyte or macrophagederived cytokines are among the most potent mediators of inflammation, involved in upregulation of osteoclasts formation, activation, and signaling pathways [36].

Osteoclasts progenitors are recruited under physiological conditions from the haematopoyetic system and can get stimulated by some cytokines and hormones to form precursors that will eventually express cell surface markers of a welldifferentiated osteoclast; furthermore, some inflammatory synovial cells, present in RA, particularly mononuclear cells and giant multinucleated cells can be differentiated into osteoclasts [5], under proinflammatory cytokines stimuli, including tumor necrosis factor alpha (TNF- $\alpha$ ), interleukin 1 (IL-1), and interleukin 6 (IL-6) [6].

Recently Pathak et al. described that sera from RA active patients contribute to bone loss by two mechanisms: (1) directly inhibiting osteoblasts proliferation and differentiation and (2) enhancing osteoblast-mediated osteoclastogenesis via RANKL and IL-6 [37].

Another key component that has influence over the osteoclastic differentiation is RANK, a receptor for TNF, attached to the membrane of osteoclast precursor cells that can bind to RNAKL through interaction with osteoblastic stromal cells $[9,38,39]$. RANK is essential for signal transduction that will lead to ostoclastic differentiation. Osteoprotegerin (OPG) also known as inhibition factor for osteoclastogenesis is a member of the TNFRSF, located in chromosome 8q24 [17] with a length of $29 \mathrm{~Kb}$ [13]; it is synthesized originally as a propeptide of $400 \mathrm{aa}$ and is secreted as a soluble protein after the cleavage of 21 aa that corresponds to the transmembrane and cytoplasmic domains [17]. OPG mRNA is expressed in different human tissues (lung, heart, kidney, liver, intestines, stomach, cerebrum, thyroid gland, and bone marrow) apart from the bone, where its primary function appears to be inhibition of osteoclasts maturation and activation. In this study, we evaluated the association of three TNFRSF11B promoter 
TABLE 3: Genotype and allele frequencies of polymorphisms in the TNFRSF11B (OPG) gene in healthy subjects and rheumatoid arthritis (RA) patients.

\begin{tabular}{|c|c|c|c|c|c|c|c|c|}
\hline SNP & Genotypes & HS & RA & Allele & HS & RA & $P$ & $\begin{array}{c}\text { OR } \\
(95 \% \mathrm{CI})\end{array}$ \\
\hline \multirow{3}{*}{$\begin{array}{l}\text { C950T } \\
\text { rs2073617 }\end{array}$} & $\mathrm{CC}$ & $9(17.6)$ & $28(43.1)$ & $\mathrm{C}$ & $47(46.1)$ & $82(63.1)$ & \multirow{3}{*}{0.01} & \multirow{3}{*}{$0.50(0.30-0.85)$} \\
\hline & CT & $29(56.9)$ & $26(40)$ & & & & & \\
\hline & $\mathrm{TT}$ & $13(25.5)$ & $11(16.9)$ & 1 & $55(53.9)$ & $48(36.9)$ & & \\
\hline \multirow{3}{*}{$\begin{array}{l}\text { C209T } \\
\text { rs3134070 }\end{array}$} & $\mathrm{CC}$ & $39(75.0)$ & $52(72.2)$ & $\mathrm{C}$ & $89(85.6)$ & $122(84.7)$ & \multirow{3}{*}{1.0} & \multirow{3}{*}{$1.1(0.53-2.2)$} \\
\hline & $\mathrm{CT}$ & $11(21.2)$ & $18(25.0)$ & $\mathrm{T}$ & $15(14.4)$ & & & \\
\hline & $\mathrm{TT}$ & $2(3.8)$ & $2(2.8)$ & 1 & $15(14.4)$ & $22(15.3)$ & & \\
\hline \multirow{3}{*}{$\begin{array}{l}\text { T245G } \\
\text { rs3134069 }\end{array}$} & $\mathrm{AA}$ & $41(78.8)$ & $66(85.7)$ & A & $93(89.4)$ & $142(92.2)$ & \multirow{3}{*}{0.51} & \multirow{3}{*}{$0.71(0.30-1.69)$} \\
\hline & $\mathrm{AC}$ & $11(21.2)$ & $10(13)$ & $\mathrm{C}$ & & & & \\
\hline & $\mathrm{CC}$ & $0(0)$ & $1(1.3)$ & C & $11(10.0)$ & $12(1.8)$ & & \\
\hline
\end{tabular}

OPG: osteoprotegerin, SNP: single nucleotide polymorphism, RA: rheumatoid arthritis, HS: healthy subjects, OR: odds ratios, and 95\% CI: 95\% confidence intervals. In order to compute OR $(95 \% \mathrm{CI})$ the following alleles were used as reference: in correspondence with the allele of risk: rs $2073617 \mathrm{~T}$, rs $3134070 \mathrm{~T}$, and rs3134069 C.

TABLE 4: Genotype and allele frequencies of polymorphisms in the TNFRSF11B (OPG) gene in rheumatoid arthritis (RA) patients with normal and low bone mineral density (BMD).

\begin{tabular}{|c|c|c|c|c|c|c|c|c|}
\hline SNP & Genotypes & $\begin{array}{l}\text { RA with } \\
\text { normal BMD }\end{array}$ & $\begin{array}{l}\text { RA with low } \\
\text { BMD }\end{array}$ & Allele & $\begin{array}{l}\text { RA with } \\
\text { normal BMD }\end{array}$ & RA with low BMD & $P$ & $\begin{array}{c}\text { OR } \\
(95 \% \mathrm{CI})\end{array}$ \\
\hline \multirow{3}{*}{$\begin{array}{l}\text { C950T } \\
\text { rs2073617 }\end{array}$} & $\mathrm{CC}$ & $9(50.0)$ & $16(44.4)$ & $\mathrm{C}$ & $25(69.4)$ & $46(63.9)$ & \multirow{3}{*}{0.67} & \multirow{3}{*}{$1.3(0.55-3.03)$} \\
\hline & CT & $7(38.9)$ & $14(38.9)$ & $\mathrm{T}$ & & & & \\
\hline & TT & $2(11.1)$ & $6(16.7)$ & 1 & $11(30.6)$ & $20(30.1)$ & & \\
\hline \multirow{3}{*}{$\begin{array}{l}\mathrm{C} 209 \mathrm{~T} \\
\text { rs3134070 }\end{array}$} & $\mathrm{CC}$ & $17(81.0)$ & $29(69.0)$ & $\mathrm{C}$ & $37(88.1)$ & $70(83.3)$ & \multirow{3}{*}{0.60} & \multirow{3}{*}{$1.5(0.49-4.43)$} \\
\hline & CT & $3(14.3)$ & $12(28.6)$ & $\mathrm{T}$ & $5(119)$ & $14(16.7)$ & & \\
\hline & TT & $1(4.8)$ & $1(2.4)$ & & & $14(10.7)$ & & \\
\hline \multirow{3}{*}{$\begin{array}{l}\text { T245G } \\
\text { rs3134069 }\end{array}$} & AA & $20(90.9)$ & $39(88.6)$ & $\mathrm{A}$ & $42(95.5)$ & $82(93.2)$ & \multirow{3}{*}{0.72} & \multirow{3}{*}{$1.5(0.29-7.95)$} \\
\hline & $\mathrm{AC}$ & $2(9.1)$ & $4(9.1)$ & C & & & & \\
\hline & $\mathrm{CC}$ & $0(0)$ & $1(2.3)$ & C & ) & $0.0)$ & & \\
\hline
\end{tabular}

OPG: osteoprotegerin, SNP: single nucleotide polymorphism, RA: rheumatoid arthritis, BMD: bone mineral density, OR: odds ratios, and 95\% CI: 95\% confidence intervals. In order to compute OR $(95 \% \mathrm{CI})$ the following alleles were used as reference: in correspondence with the allele of risk: rs $2073617 \mathrm{~T}$, rs3134070 T, and rs3134069 C.

polymorphisms with presence of osteoporosis in RA. Our study demonstrated that the $\mathrm{C}$ allele of the C950 T was more frequent in RA compared with HS; thereafter we analyzed the RA group to seek for differences in allele distribution between patients with and without osteoporosis; nevertheless we did not observe a significant difference. The other two polymorphisms did not reach statistical significance.

The observed association in the present study could be caused by direct functional effect of the SNP or to linkage disequilibrium (LD) with another functional variant. According to the HapMap data, there is a certain amount of LD between these SNPs, indicating that although it is possible that different causative variants exist in TNFRSF11B, it is also possible that only one of them turns out to have functional consequences. The C950T SNP is located in the promoter region, near the TGF- $\beta$ response area and $129 \mathrm{bp}$ upstream the TATA box [40]. A functional study of TNFRSF11B polymorphisms through luciferase reporter assays revealed that gene expression was affected by the C950T polymorphism, since it was significantly higher in the presence of the $\mathrm{C}$ allele [27].

Previous association studies that include polymorphisms in the TNFRSF11B gene were performed in osteoporotic patients. In 2002 Ohmori et al. showed a significant association between C950T SNP and lower BMD [25]; in contrast with such results in 2004 Brändström et al. showed that C950T was not associated with bone mineral density in elderly Swedish women [41], and later Arko et al. in 2005 tested the three polymorphisms included in the present study and others searching for an association with bone mineral density in postmenopausal women; the linkage disequilibrium was confirmed but no associations were found [26]. For SNP T245G, similar frequencies as obtained in this study have been described previously by Zajíčková et al. in postmenopausal women [42]. Only a few studies have looked for an association between these SNPs and RA; in 2010 Assmann et al. performed a case-control study and found an association between SNPs in the RANK and RANKL gene but 
not in the OPG gene; nevertheless none of the SNPs included in our study were genotyped by them [43]. In a recent study the SNP A163G in the osteoprotegerin gene was associated with osteoporosis in RA [44]. This polymorphism was not included in the present study but a lack of association with this particular polymorphism and osteoporosis in RA was previously confirmed by our study group [45].

Because this work constitutes a preliminary report, a limitation in our study is a small sample size that may limit our statistical power to detect small differences between groups although we consider that our sample would be sufficient to identify polymorphisms that may confer a high risk for low BMD in RA and did not show any trend to suspect that increasing the sample size a statistical significance would be achieved. We recognize that future multicenter studies should be performed, increasing the sample size, with our results being useful as a referent for Mexican patients with RA to identify that these polymorphisms probably do not confer a high risk for low BMD in our population with RA. Further studies in other populations are required to confirm our findings.

In conclusion, we confirmed the association of $\mathrm{C}$ allele of the C950T SNP in the TNFRSF11B gene with RA patients, but we were not able to support our main hypothesis that this polymorphism in the OPG gene is associated with osteoporosis in RA. Further studies including other regions of Mexico and Latin America with an increased sample size are required to confirm our findings and further elucidate if C950T SNP in the OPG gene could be associated with osteoporosis and not only with RA presence.

\section{Ethical Approval}

This research was conducted in accordance with the Declaration of Helsinki. Informed consent was obtained from all participants, the study protocol was approved by the Local Committees for Ethical and Health Research (CLIEIS 20071301-1), and all data was managed anonymously.

\section{Conflict of Interests}

The authors declare that there is no conflict of interests regarding the publication of this paper.

\section{Acknowledgment}

This work was supported by Grant from Instituto Mexicano del Seguro Social through FIS/IMSS/PROT/C2007/025.

\section{References}

[1] M. Akil and R. S. Amos, "Rheumatoid arthritis, I: clinical features and diagnosis," British Medical Journal, vol. 310, no. 6979, pp. 587-590, 1995.

[2] M. H. Cardiel and J. Rojas-Serrano, "Community based study to estimate prevalence, burden of illness and help seeking behavior in rheumatic diseases in Mexico City. A COPCORD study," Clinical and Experimental Rheumatology, vol. 20, no. 5, pp. 617624, 2002.
[3] J. A. Kanis, L. J. Melton III, C. Christiansen, C. C. Johnston, and N. Khaltaev, "The diagnosis of osteoporosis," Journal of Bone and Mineral Research, vol. 9, no. 8, pp. 1137-1141, 1994.

[4] F. Alcaraz-Lopez Miriam, G.-L. L. Aguilar-Chavez Erika, A. Lopez-Olivo Maria, C. Loaiza-Cardenas, and I. Gamez-Nava Jorge, "Performance of albrand index for identifying low bone density in rheumatoid arthritis," The Journal of Rheumatology, vol. 33, no. 2, p. 408, 2006.

[5] E. M. Gravallese, "Bone destruction in arthritis," Annals of the Rheumatic Diseases, vol. 61, supplement 2, pp. ii84-ii86, 2002.

[6] S. Roux and P. Orcel, "Bone loss: factors that regulate osteoclast differentiation: an update," Arthritis Research, vol. 2, no. 6, pp. 451-456, 2000.

[7] D. L. Lacey, E. Timms, H.-L. Tan et al., "Osteoprotegerin ligand is a cytokine that regulates osteoclast differentiation and activation," Cell, vol. 93, no. 2, pp. 165-176, 1998.

[8] N. Udagawa, N. Takahashi, H. Yasuda et al., "Osteoprotegerin produced by osteoblasts is an important regulator in osteoclast development and function," Endocrinology, vol. 141, no. 9, pp. 3478-3484, 2000.

[9] H. Hsu, D. L. Lacey, C. R. Dunstan et al., "Tumor necrosis factor receptor family member RANK mediates osteoclast differentiation and activation induced by osteoprotegerin ligand," Proceedings of the National Academy of Sciences of the United States of America, vol. 96, no. 7, pp. 3540-3545, 1999.

[10] M. C. Bezerra, J. F. Carvalho, A. S. Prokopowitsch, and R. M. R. Pereira, "RANK, RANKL and osteoprotegerin in arthritic bone loss," Brazilian Journal of Medical and Biological Research, vol. 38, no. 2, pp. 161-170, 2005.

[11] D. H. Jones, Y.-Y. Kong, and J. M. Penninger, "Role of RANKL and RANK in bone loss and arthritis," Annals of the Rheumatic Diseases, vol. 61, supplement 2, pp. ii32-ii39, 2002.

[12] S.-S. Dong, X.-G. Liu, Y. Chen et al., "Association analyses of RANKL/RANK/OPG gene polymorphisms with femoral neck compression strength index variation in caucasians," Calcified Tissue International, vol. 85, no. 2, pp. 104-112, 2009.

[13] Y.-H. Hsu, T. Niu, H. A. Terwedow et al., "Variation in genes involved in the RANKL/RANK/OPG bone remodeling pathway are associated with bone mineral density at different skeletal sites in men," Human Genetics, vol. 118, no. 5, pp. 568-577, 2006.

[14] C. Liu, T. S. Walter, P. Huang et al., "Structural and functional insights of RANKL-RANK interaction and signaling," Journal of Immunology, vol. 184, no. 12, pp. 6910-6919, 2010.

[15] A.-P. Trouvin and V. Goëb, "Receptor activator of nuclear factor- $\kappa \mathrm{B}$ ligand and osteoprotegerin: maintaining the balance to prevent bone loss," Clinical Interventions in Aging, vol. 5, pp. 345-354, 2010.

[16] J. B. Richards, F. K. Kavvoura, F. Rivadeneira et al., "Collaborative meta-analysis: associations of 150 candidate genes with osteoporosis and osteoporotic fracture," Annals of Internal Medicine, vol. 151, no. 8, pp. 528-537, 2009.

[17] P. Narducci, R. Bareggi, and V. Nicolin, "Receptor Activator for Nuclear Factor kappa B Ligand (RANKL) as an osteoimmune key regulator in bone physiology and pathology," Acta Histochemica, vol. 113, no. 2, pp. 73-81, 2011.

[18] W. J. Boyle, W. S. Simonet, and D. L. Lacey, "Osteoclast differentiation and activation," Nature, vol. 423, no. 6937, pp. 337-342, 2003.

[19] B. Arko, J. Preelj, R. Komel, A. Kocijani, P. Hudler, and J. Marc, "Sequence variations in the osteoprotegerin gene promoter in patients with postmenopausal osteoporosis," Journal of Clinical 
Endocrinology and Metabolism, vol. 87, no. 9, pp. 4080-4084, 2002.

[20] F. C. Arnett, S. M. Edworthy, D. A. Bloch et al., "The American Rheumatism Association 1987 revised criteria for the classification of rheumatoid arthritis," Arthritis and Rheumatism, vol. 31, no. 3, pp. 315-324, 1988.

[21] M. L. L. Prevoo, M. A. van'T Hof, H. H. Kuper, M. A. van Leeuwen, L. B. A. van de Putte, and P. L. C. M. van Riel, "Modified disease activity scores that include twenty-eight-joint counts development and validation in a prospective longitudinal study of patients with rheumatoid arthritis," Arthritis \& Rheumatism, vol. 38, no. 1, pp. 44-48, 1995.

[22] M. H. Cardiel, M. Abello-Banfi, R. Ruiz-Mercado, and D. Alarcon-Segovia, "How to measure health status in rheumatoid arthritis in non-English speaking patients: validation of a Spanish version of the Health Assessment Questionnaire Disability Index (Spanish HAQ-DI)," Clinical and Experimental Rheumatology, vol. 11, no. 2, pp. 117-121, 1993.

[23] M. C. Hochberg, R. W. Chang, I. Dwosh, S. Lindsey, T. Pincus, and F. Wolfe, "The American College of Rheumatology 1991 revised criteria for the classification of global functional status in rheumatoid arthritis," Arthritis and Rheumatism, vol. 35, no. 5, pp. 498-502, 1992.

[24] S. Gustincich, G. Manfioletti, G. del Sal, C. Schneider, and P. Carninci, "A fast method for high-quality genomic DNA extraction from whole human blood," BioTechniques, vol. 11, no. 3, pp. 298-302, 1991.

[25] H. Ohmori, Y. Makita, M. Funamizu et al., "Linkage and association analyses of the osteoprotegerin gene locus with human osteoporosis," Journal of Human Genetics, vol. 47, no. 8, pp. 400-406, 2002.

[26] B. Arko, J. Preželj, A. Kocijančič, R. Komel, and J. Marc, "Association of the osteoprotegerin gene polymorphisms with bone mineral density in postmenopausal women," Maturitas, vol. 51, no. 3, pp. 270-279, 2005.

[27] C. Vidal, R. Formosa, and A. Xuereb-Anastasi, "Functional polymorphisms within the TNFRSF11B (osteoprotegerin) gene increase the risk for low bone mineral density," Journal of Molecular Endocrinology, vol. 47, no. 3, pp. 327-333, 2011.

[28] G. Beyens, A. Daroszewska, F. de Freitas et al., "Identification of sex-specific associations between polymorphisms of the Osteoprotegerin gene, TNFRSF11B, and Paget's disease of bone," Journal of Bone and Mineral Research, vol. 22, no. 7, pp. 10621071, 2007.

[29] M. Soufi, M. Schoppet, A. M. Sattler et al., "Osteoprotegerin gene polymorphisms in men with coronary artery disease," Journal of Clinical Endocrinology and Metabolism, vol. 89, no. 8, pp. 3764-3768, 2004.

[30] D. Pitocco, G. Zelano, G. Gioffrè et al., "Association between osteoprotegerin G1181C and T245G polymorphisms and diabetic charcot neuroarthropathy: a case-control study," Diabetes Care, vol. 32, no. 9, pp. 1694-1697, 2009.

[31] C. Cooper, G. Campion, and L. J. Melton III, "Hip fractures in the elderly: a world-wide projection," Osteoporosis International, vol. 2, no. 6, pp. 285-289, 1992.

[32] S. Y. Kim, S. Schneeweiss, J. Liu et al., "Risk of osteoporotic fracture in a large population-based cohort of patients with rheumatoid arthritis," Arthritis Research and Therapy, vol. 12, no. 4, article R154, 2010.

[33] S. R. Goldring, "The final pathogenetic steps in focal bone erosions in rheumatoid arthritis," Annals of the Rheumatic Diseases, vol. 59, no. 1, pp. i72-i74, 2000.
[34] R. M. R. Pereira, J. F. de Carvalho, and E. Canalis, "Glucocorticoid-induced osteoporosis in rheumatic diseases," Clinics, vol. 65, no. 11, pp. 1197-1205, 2010.

[35] P. Geusens and W. F. Lems, "Osteoimmunology and osteoporosis," Arthritis Research and Therapy, vol. 13, no. 5, article 242, 2011.

[36] G. Mori, P. D’Amelio, R. Faccio, and G. Brunetti, “The interplay between the bone and the immune system," Clinical and Developmental Immunology, vol. 2013, Article ID 720504, 16 pages, 2013.

[37] J. L. Pathak, N. Bravenboer, P. Verschueren et al., "Inflammatory factors in the circulation of patients with active rheumatoid arthritis stimulate osteoclastogenesis via endogenous cytokine production by osteoblasts," Osteoporosis International, vol. 25, no. 10, pp. 2453-2463, 2014.

[38] J. Li, I. Sarosi, X.-Q. Yan et al., "RANK is the intrinsic hematopoietic cell surface receptor that controls osteoclastogenesis and regulation of bone mass and calcium metabolism," Proceedings of the National Academy of Sciences of the United States of America, vol. 97, no. 4, pp. 1566-1571, 2000.

[39] T. L. Burgess, Y.-X. Qian, S. Kaufman et al., "The ligand for osteoprotegerin (OPGL) directly activates mature osteoclasts," Journal of Cell Biology, vol. 145, no. 3, pp. 527-538, 1999.

[40] K. Thirunavukkarasu, R. R. Miles, D. L. Halladay et al., "Stimulation of osteoprotegerin (OPG) gene expression by transforming growth factor- $\beta$ (TGF- $\beta$ ). Mapping of the OPG promoter region that mediates TGF- $\beta$ effects," The Journal of Biological Chemistry, vol. 276, no. 39, pp. 36241-36250, 2001.

[41] H. Brändström, P. Gerdhem, F. Stiger et al., "Single nucleotide polymorphisms in the human gene for osteoprotegerin are not related to bone mineral density or fracture in elderly women," Calcified Tissue International, vol. 74, no. 1, pp. 18-24, 2004.

[42] K. Zajíčková, A. Zemanová, M. Hill, and I. Žofková, "Is A163G polymorphism in the osteoprotegerin gene associated with heel velocity of sound in postmenopausal women?" Physiological Research, vol. 57, no. 1, pp. S153-S157, 2008.

[43] G. Assmann, J. Koenig, M. Pfreundschuh et al., "Genetic variations in genes encoding RANK, RANKL, and OPG in rheumatoid arthritis: a case-control study," Journal of Rheumatology, vol. 37, no. 5, pp. 900-904, 2010.

[44] Y. M. Hussien, A. Shehata, R. A. Karam, S. S. Alzahrani, H. Magdy, and A. M. El-Shafey, "Polymorphism in vitamin D receptor and osteoprotegerin genes in Egyptian rheumatoid arthritis patients with and without osteoporosis," Molecular Biology Reports, vol. 40, no. 5, pp. 3675-3680, 2013.

[45] A. J. L. Brambila-Tapia, J. Durán-González, L. SandovalRamírez et al., "MTHFR C677T, MTHFR A1298C, and OPG A163G polymorphisms in Mexican patients with rheumatoid arthritis and osteoporosis," Disease Markers, vol. 32, no. 2, pp. 109-114, 2012. 


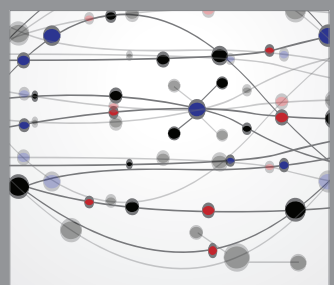

The Scientific World Journal
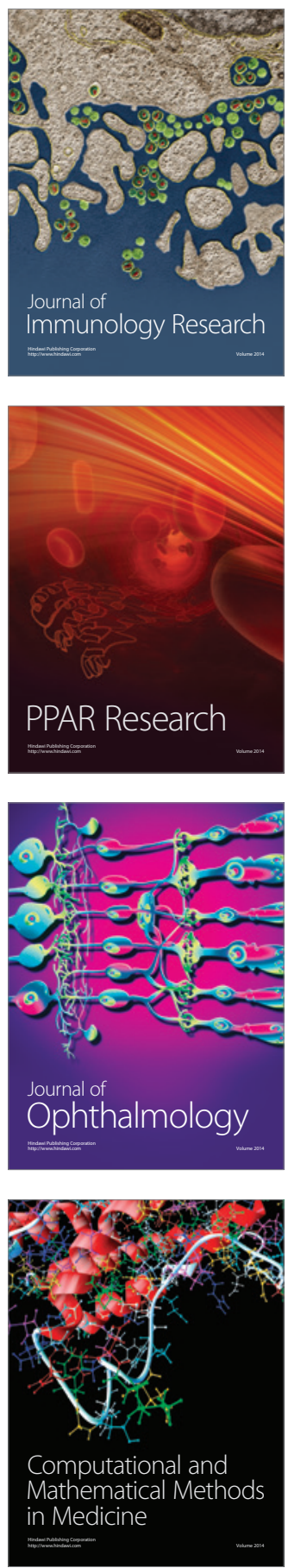

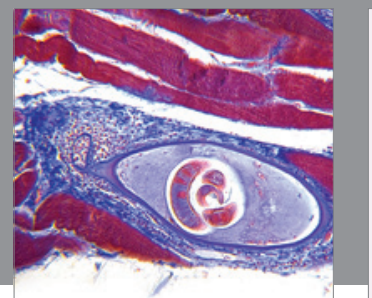

Gastroenterology

Research and Practice
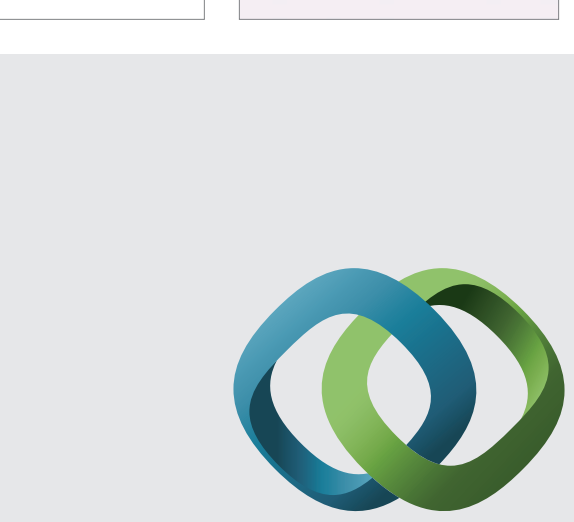

\section{Hindawi}

Submit your manuscripts at

http://www.hindawi.com
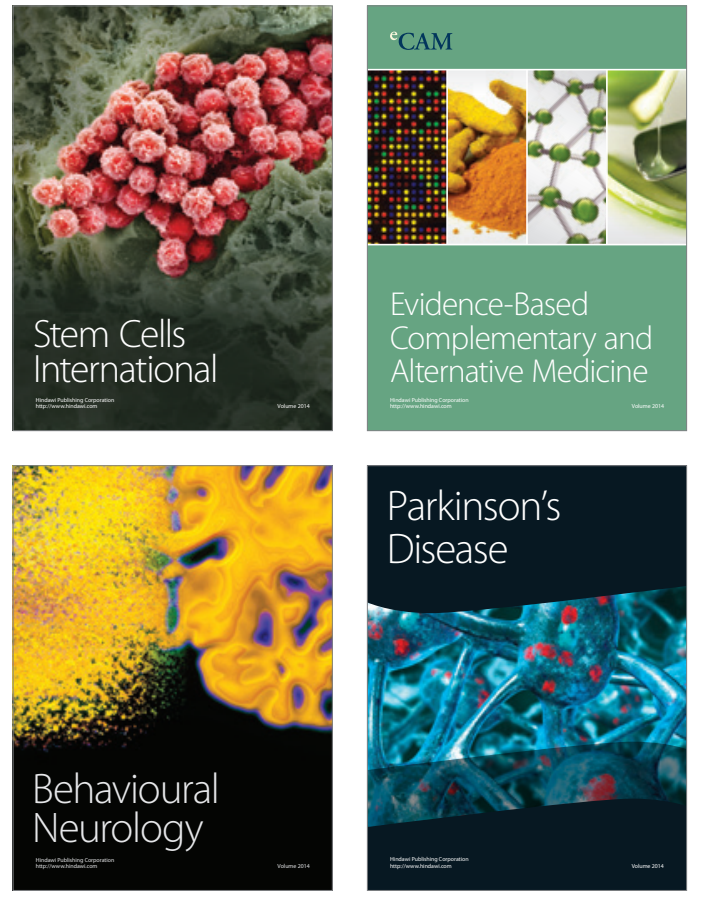
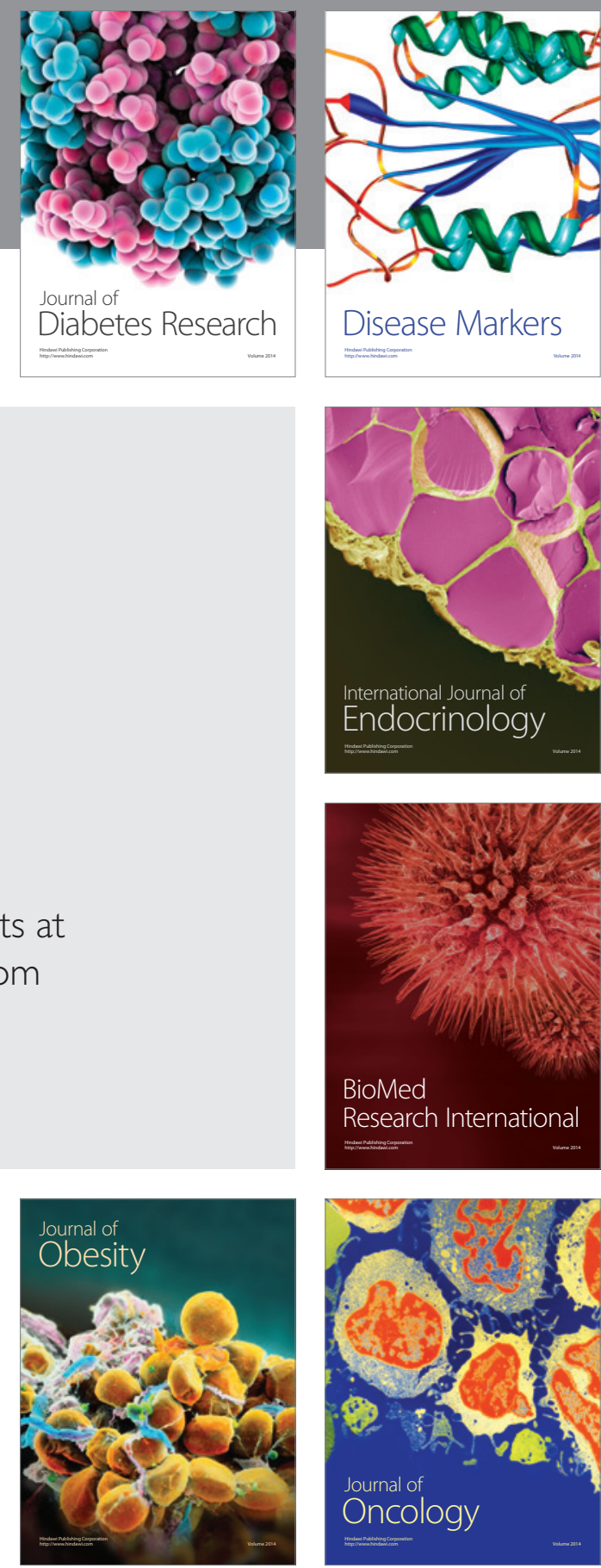

Disease Markers
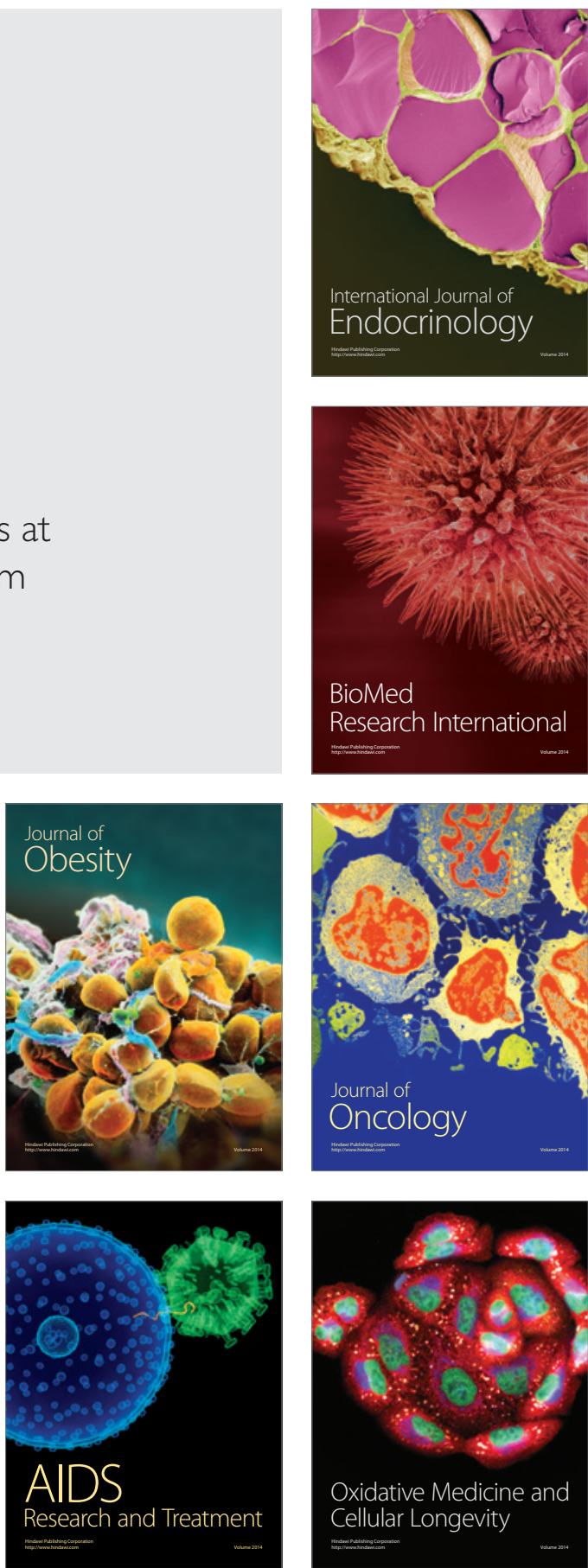